\title{
CONFECTIO GARI POMPEIANI. PROCEDIMIENTO EXPERIMENTAL PARA LA ELABORACIÓN DE SALSAS DE PESCADO ROMANAS
}

\author{
CONFECTIO GARI POMPEIANI. EXPERIMENTAL PROCEDURE \\ FOR THE PREPARATION OF ROMAN FISH SAUCES
}

\author{
ENRIQUE GARCÍA VARGAS* \\ DARÍO BERNAL CASASOLA** \\ VÍCTOR PALACIOS MACÍAS*** \\ ANA MARÍA ROLDÁN GÓMEZ*** \\ ÁLVARO RODRÍGUEZ ALCÁNTARA*** \\ JOSEFINA SÁNCHEZ GARCÍA***
}

\begin{abstract}
Resumen: El presente trabajo hace públicos por primera vez los resultados de un proceso experimental fruto de la colaboración entre las universidades de Cádiz y Sevilla. Dicho proceso, en el que han participado arqueólogos y bioquímicos, se ha realizado en el Departamento de Ingeniería Química de la Facultad de Ciencias de la Universidad de Cádiz y ha consistido en la identificación de la naturaleza concreta y en la reproducción física de la afamada salsa salada de pescado greco-romana denominada garum (liquamen), de un subproducto alimenticio sólido de esta (hallec) y de una salsa secundaria y de menor calidad denominada en latín muria. El estudio filológico de antiguas recetas de confección del garum (confectio gari) y los resultados analíticos de los residuos de estos productos recogidos durante la excavación de la denominada bottega o tienda del garum de Pompeya han permitido simular su producción artesanal en laboratorio y obtener unos condimentos salados cuyo aspecto físico y características alimentarias coinciden con los descritos por las fuentes literarias grecorromanas y con los que aún muestran los residuos orgánicos analizados de estas producciones.
\end{abstract}

Palabras Clave: garum, Pompeya, proceso experimental, elaboración del garum

\footnotetext{
* Departamento de Prehistoria y Arqueología. Universidad de Sevi1la. c/ Dña. María de Padilla s/n, 41004, Sevilla. Correo-e: egarcia@us.es

** Área de Arqueología. Departamento de Historia, Geografía y Filosofía. Universidad de Cádiz. Avda. Dr. Gómez Ulla 1, 11003 Cádiz. Correo-e: dario.bernal@uca.es
}

Abstract: The present work publishes for the first time the results of an experimental process carried out in cooperation between the Universities of Cádiz and Seville. This process, involving the participation of archaeologists and experts in biochemistry, has been effected in the chemical engineering department of the University of Cádiz and has focused on the characterization and reproduction of the famous GraecoRoman salted fish sauce known as garum (liquamen), of a solid by-product of this sauce (hallec) and of a secondary, lesser quality, sauce known in Latin as muria. The philological analysis of ancient recipes (confectio gari) and the analysis of the residues collected in the so-called bottega or Garum Shop, in Pompeii, have permitted their artisanal reproduction in the laboratory. The physical aspect and alimentary features of these products coincide with those described in the ancient sources and with the organic residues under analysis.

Key words: garum, Pompeii, experimental process, garum production 


\section{OBJETIVOS Y METODOLOGÍA}

El objetivo fundamental del presente trabajo es hacer públicos los resultados de un experimento realizado en colaboración entre las universidades de Cádiz y Sevilla que ha tenido por resultado la formalización de un procedimiento muy próximo al artesanal romano para la confección de una salsa de pescado de tipo liquamen, así como de dos de sus derivados alimenticios (la muria y el hallec). El aspecto físico y las propiedades bioquímicas de estos productos han servido, a su vez, como referentes para la determinación del tipo de producto al que correspondía el sedimento orgánico recogido del interior de cinco dolia localizados en el patio de la casa pompeyana denominada "La bottega del garum", ya que se trata de un establecimiento de elaboración de salazones y salsas de pescado (Curtis 1979) en el que algunos de los firmantes (Bernal, Cottica y Zaccaria 2009 y 2010; Bernal Casasola et alii 2009 y 2013) se encuentran trabajando en el seno del proyecto internacional El Garum de Pompeya y Herculano. Explotación de los recursos del mar en el ámbito vesubiano.

En este trabajo han colaborado los departamentos de Ingeniería Química y Tecnología de los Alimentos, e Historia, Geografía y Filosofía de la Universidad de Cádiz, así como el de Prehistoria y Arqueología de la Universidad de Sevilla.

La metodología de obtención del liquamen se ha basado en tres procedimientos básicos, siendo uno de ellos filológico y los otros dos de carácter técnico:

- El procedimiento filológico ha consistido en la elección de una receta histórica con información suficiente sobre los ingredientes utilizados, las proporciones de los mismos y los pasos a seguir durante el proceso de confección del liquamen;

- El primer procedimiento técnico ha sido la obtención en el laboratorio del Departamento de Ingeniería Química y Tecnología de los Alimentos de la Universidad de Cádiz de dicho producto (liquamen) y sus subproductos o derivados (una salsa de menor calidad que sobrenada el producto en los primeros momentos de la confección, la muria, y un residuo formado por elementos no hidrolizados, especialmente de carácter óseo, que consideramos equivale al hallex de las fuentes literarias romanas), en condiciones que simulan las del procedimiento artesanal descrito por las fuentes documentales.

- Finalmente, el segundo procedimiento técnico realizado en el mismo laboratorio ha consistido en el análisis físico-químico de los residuos de pescado procedentes de contextos arqueológicos controlados, en este caso los seis dolia o grandes contenedores conservados en el Ambiente 9 de la llamada Bottega del Garum de Pompeya (I, 12, 8), donde según las investigaciones en curso se habrían elaborado salsas similares a las descritas en las fuentes y reproducidas por nosotros en laboratorio (fig. 1). El objeto de esta analítica de residuos es contrastar las características físico-químicas de los residuos de salsas de pescado romanas extraídas de los dolia pompeyanos con las del producto obtenido en laboratorio.

\section{EL PROCEDIMIENTO FILOLÓGICO: ELECCIÓN DE LA RECETA}

La literatura moderna acerca de los distintos tipos y calidades de salsas y salazones de pescado romanas es ya inmensa (cf. Curtis 1991; Etienne y Mayet 2002). Sin embargo, desde principios del siglo XIX (Köhler 1832) se ha dedicado en lo sustancial a la misma $y$ compleja tarea: determinar los distintos productos y sus formas de preparación a partir de los datos de la evidencia literaria antigua, de analogías etnográficas con producciones similares tradicionales del Sudeste asiático (Grimal y Monod 1952, Jardin 1961) y de los restos de producto hallados en recipientes arqueológicos, ya sean de elaboración (piletas de salazón, grandes contenedores tipo dolium), transporte (ánforas) o servicio (jarras, orzas etc.).

La dificultad radica en la falta de concreción de la evidencia literaria acerca de las formas y los procesos de elaboración concretos para cada producto de los citados que, dado lo escueto de la información escrita, son difíciles de reconocer en restos orgánicos de tipo arqueológico que han llegado hasta nuestros días bastante degradados. Sobre la base de la evidencia escrita (tradición literaria más rótulos pintados sobre las ánforas salsarias) se reconocen cuatro preparados cuyo carácter general está más o menos claro, pero cuya determinación y caracterización exacta está lejos de ser admitida por todos los investigadores: el garum, la muria, el liquamen y el hallec, pudiendo este último encontrarse escrito también como allec, hallex o allex.

Todos los preparados citados tienen en común estar constituidos por salsas saladas de pescado que se diferencian claramente de los pescados completos o de los trozos de pescado conservados en sal -salazones o 


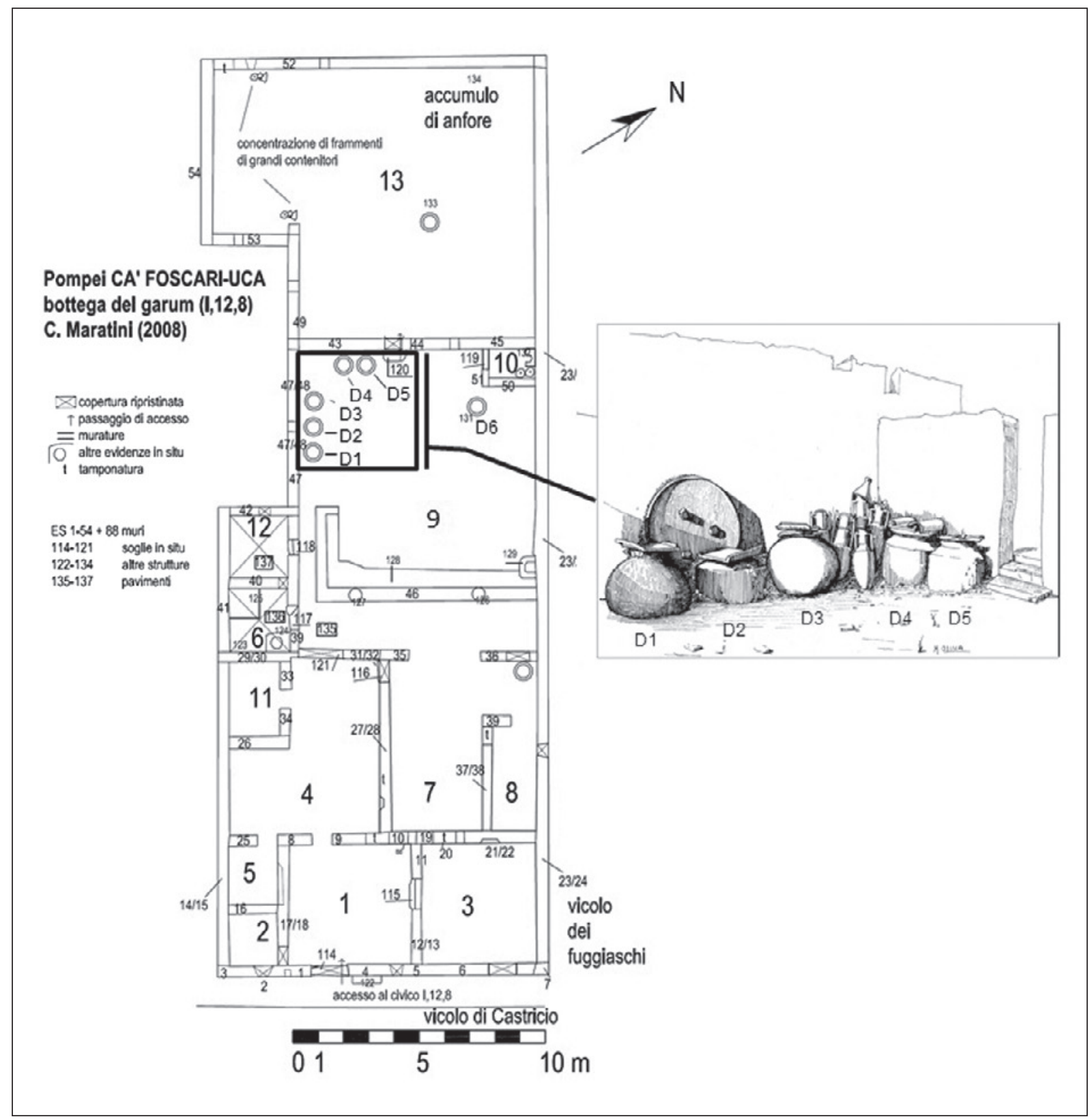

Figura 1. Planimetría de la Bottega del Garum de Pompeya con la ubicación de los dolia objeto de estudio.

salsamenta- (García Vargas y Bernal 2009). Para obtener una salsa de pescado es necesario partir de pescados salados (completos o cortados en trozos, según el tamaño del pez) que sometidos a un proceso bioquímico de autolisis enzimática, se licuan literalmente, dando lugar a una salsa salada más o menos líquida, más o menos pastosa, que se usaba normalmente como condimento de otros platos. Para que se produzca la hidrólisis es imprescindible la presencia en el proceso de los jugos gástricos del pescado, pues, en palabras de P. Grimal y Th. Monod, se trata de la «macération de certains poissons dans une saumure concentrée de sel marin; ceux-ci, sous l'influence de la température et dans un milieu aseptique, se digèrent eux-mêmes par une histolyse de leurs tissus musculaires en présence et grâce aux diastases contenues dans leur propre tube digestifi(Grimal y Monod 1952, 31). Esto quiere decir que para la confección de salsas de pescado es 
imprescindible incluir en el preparado inicial, de una $\mathrm{u}$ otra manera, el tracto digestivo de los peces.

Con peces de gran tamaño (atún, bonito, caballa), era habitual utilizar como ingredientes las vísceras del animal junto a la sangre y las agallas para confeccionar las salsas, mientras que la carne limpia y troceada se preparaba en salazón (salsamentum). Si se empleaban peces de tamaño más pequeño (boquerones, sardinas) éstos se utilizaban completos y daban lugar a un producto que en principio se denominó en latín con un sustantivo genérico para los líquidos, $l i$ quamen, pero que hacia fines del siglo I d.C. se había ya especializado en la denominación de las salsas de pescado. A partir del siglo III d.C. es frecuente encontrar garum y liquamen usados como sinónimos para designar cualquier salsa de pescado de tipo garum independientemente del tamaño del pez de que procediera, puesto que, en lo sustancial, los procedimientos bioquímicos implicados en la obtención del producto eran los mismos: carne de pescado hidrolizada en presencia de sal. No obstante, el garum obtenido del atún rojo se siguió considerando el más exquisito y el de mayor calidad, razón por la que se lo distinguía a menudo con un adjetivo como haimation o hematitou (de sangre) y, más frecuentemente, sociorum o "de los aliados", haciendo referencia a la procedencia del garum más apreciado: la ciudad de Cádiz que, en origen, fue aliada o socia de la República romana (Millán León 2001).

El hallec, hallex, allec o allex es con claridad el "residuo" del garum y/o del liquamen. Esto es lo que afirma explícitamente Plinio (Nat. 37.44: uitium huius est alex, imperfecta nec colata faex), lo que significa que era necesario colar el garum para eliminar los trozos incompletamente licuados y otros residuos sólidos del producto, para lo cual existían embudos específicos que han sido localizados e identificados arqueométricamente en el registro arqueológico (Bernal y Sáez 2006). El procedimiento del filtrado o colado del garum está presente en un buen número de recetas de cronologías muy diversas (infra) y parece ser el que establece propiamente la diferencia entre el garum y/o liquamen y el subproducto llamado hallec.

La muria es el más enigmático de los productos del garum. Con carácter general, se denominaba $m u$ ria o halmé a una solución salina, a una salmuera. Cuando aparece mencionada como una salsa de pescado, se hace siempre referencia, sin embargo, a algo más que a sal disuelta en agua. Una primera indicación acerca del carácter de esta salsa la ofrece Columella (D.r.r. 12.55.4) cuando, tras explicar con detalle el procedimiento de salado de la carne de cerdo, añade que ésta se puede emplear siempre, porque "se conserva en su propia muria como la salazón [de pescado]" (tanquam salsamentum in muria sua permanet). Se deduce de ello que la muria es la salmuera compuesta por la sal empleada en el proceso de salazón disuelta en la sangre, el suero y los jugos orgánicos de la carne de cerdo o de pescado. De hecho, la confección del garum comienza siempre por la disposición de los peces o de los trozos de peces en capas alternas con sal. Al poco tiempo, los jugos de los peces, ricos en aminoácidos, van migrando por ósmosis a la parte alta del preparado, formando una salmuera que sobrenada el producto y cuya salida a la superficie del recipiente se favorece presionando la pasta con colmos o pesos, práctica observada modernamente, por ejemplo, en los saladeros tradicionales de Vietnam para la elaboración del "nuoc mam". Este proceso de exudación de la muria se ha observado igualmente en el experimento realizado en la Facultad de Ciencias de Cádiz (fig. 2).

Para nuestro experimento, era necesario partir de una de las escasas recetas conocidas para la confección del garum o liquamen. Para más de mil años de confectio gari no se conoce sino un número reducido de textos que, de forma explícita, expliquen el procedimiento más habitual, siendo todas ellas de una fecha relativamente tardía, a excepción del pasaje citado de Junio Moderato Columela (siglo I d.C.) que, en realidad, se refiere a la salazón sólida y de carne de cerdo. De una forma resumida, casi esquemática, éstas son las "recetas" con que contamos para la determinación del procedimiento

- Gargilio Marcial, Medicina ex oleribus et pomis (siglo III d.C.). Se han conservado en la abadía de Saint-Gallen dos manuscritos (Cod. Sang. 752 y 899; Figura 3) que incluyen, junto a otros escritos latinos de diversos autores, esta obra médica de Gargilio Marcial. Ambos presentan la misma "receta" para la confección del garum titulada Confectio liquaminis quod omogarum vocant. Si no se trata de una interpolación medieval (siglo IX d.C., fecha de los códices), es el texto antiguo más extenso sobre la confección del liquamen.

- Julio Africano, Las Kestoi de este autor cristiano del siglo III d.C. contienen un texto griego que, muy brevemente, instruye sobre la confección de una imitación del garon sokkios o garum sociorum (supra). Es el único que se refiere a grandes peces, pues precisa del empleo de abdomion (o abdomina), es decir, de ventresca de atún. 


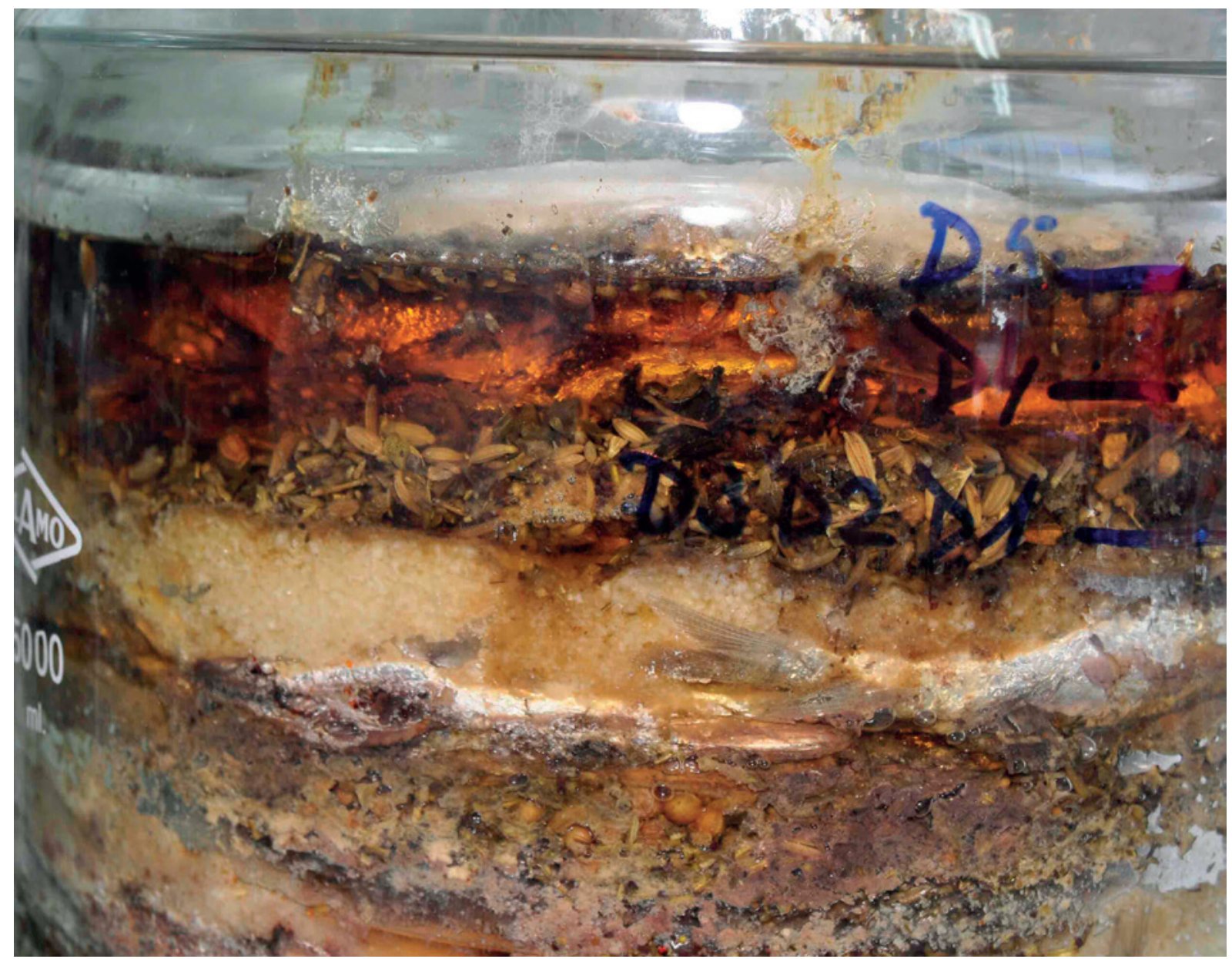

Figura 2. Detalle del líquido (muria) que sobrenada el producto en la fase estática de su elaboración.

- Manuscrito latino (Ms. Lat. No 11219) de la abadía de Echternach (Luxemburgo). Es un original altomedieval (s. IX d.C.) que contiene unas "instrucciones" para la fabricación del garum: Confectio ad garum faciendum

- Rufio Festo, Breviarium rerum gestarum populi Romani (fines del s. IV d.C.). Se encuentra interpolada en uno de los manuscritos conservados de un texto que resulta ser un resumen de historia romana redactado por un alto funcionario de la corte del emperador Valente (364-378 d.C.). Con buen criterio, se piensa que no es más que una receta (confectio gari) introducida por el copista medieval que pretendía tal vez anotarla antes de olvidarla y lo hizo en la obra sobre la que se encontraba trabajando, de cuyo texto, por tanto, no forma parte.

- Los Geoponica, tratado bizantino de agricultura que contiene un extenso (20.40.1-6) capítulo sobre la confección del garum y que ofrece diversos procedimientos para la gáron poíesis o confectio gari, al que también denomina likouamen. Data del siglo X d.C.

De todos los textos citados, el más largo, detallado y próximo teóricamente a los años del Alto Imperio Romano es el de Gargilio Marcial. Pertenece, además, a la tradición latina, la más adecuada supuestamente a los restos de producto pompeyano que teníamos como evidencia material de un producto antiguo, y se encuentra recogido en unos manuscritos cuya lectura directa es posible gracias a que se encuentran on-line en la página de la biblioteca del monasterio de Saint Gallen ${ }^{1}$. De la

1 (http://www.e-codices.unifr.ch/en/csg/0752/130/medium y http://www.e-codices.unifr.ch/en/csg/0899/139/medium, consulta 10 de junio de 2012). Para el texto original latino vid apéndice. 


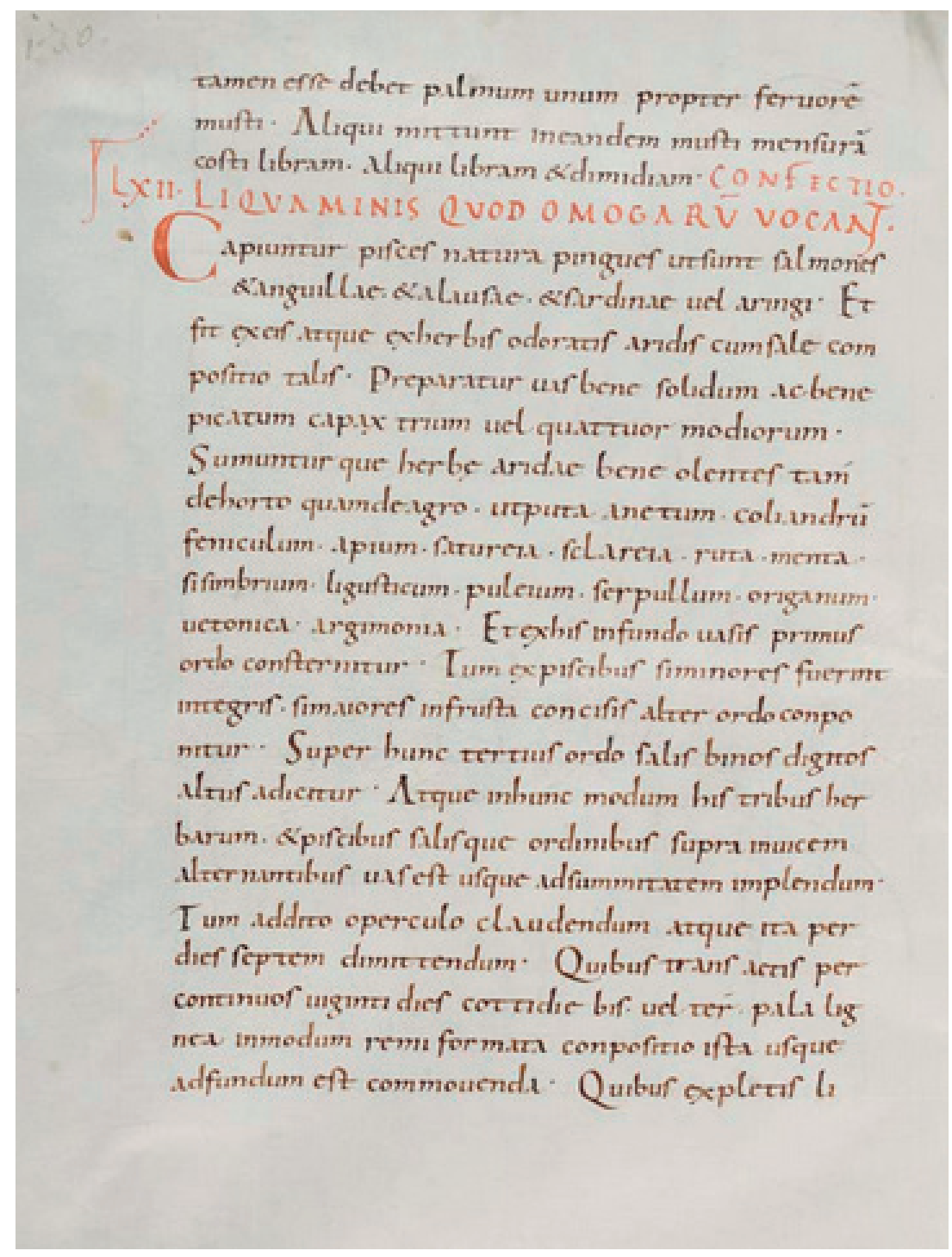

Figura 3. Inicio de la receta de Gargilius Martialis según el códice de Saint-Gallen. consulta del manuscrito original, se deduce en primer lugar que el título habitual que se le da a las recetas de Gargilio Marcial: Confectio liquaminis quod oenogarum vocant (Rose 1874, Curtis 1991) es erróneo, siendo el final correcto del mismo ...quod omogarum vocant. No se trata, por tanto, de un oenogarum o garum con vino, o no es esa la particularidad de la composición, sino de un omogarum o garum crudo, aunque pensamos que en éste caso más que crudo deberíamos traducir "simple" o rápido, porque en lugar de la maceración al sol que podía llevar hasta tres meses (cf. Geoponica 20.46.4), se acelera el proceso mediante la adición de vino, de nuevas hierbas aromáticas y la cocción de todo ello. El texto, es como sigue:

\section{«Confección del liquamen que se denomina omogarum}

Se cogen peces grasos por naturaleza, como los salmones, las anguilas, los sábalos, las sardinas y los arenques y haz con ellos la siguiente preparación: se coloca en un recipiente bien sólido y bien empegado de tres o cuatro modios de capacidad (26 a 35 litros) y se toman hierbas olorosas secas tanto cultivadas como silvestres (...). Se disponen en el fondo del recipiente formando una primera capa; después se coloca otra capa de peces, completos si fueran pequeños y cortados en trozos si fueran grandes; sobre ésta se añade otra capa de dos dedos de sal. Y, de este modo, el recipiente se llena hasta arriba con estas capas siempre alternantes de hierbas, de pescado y de 


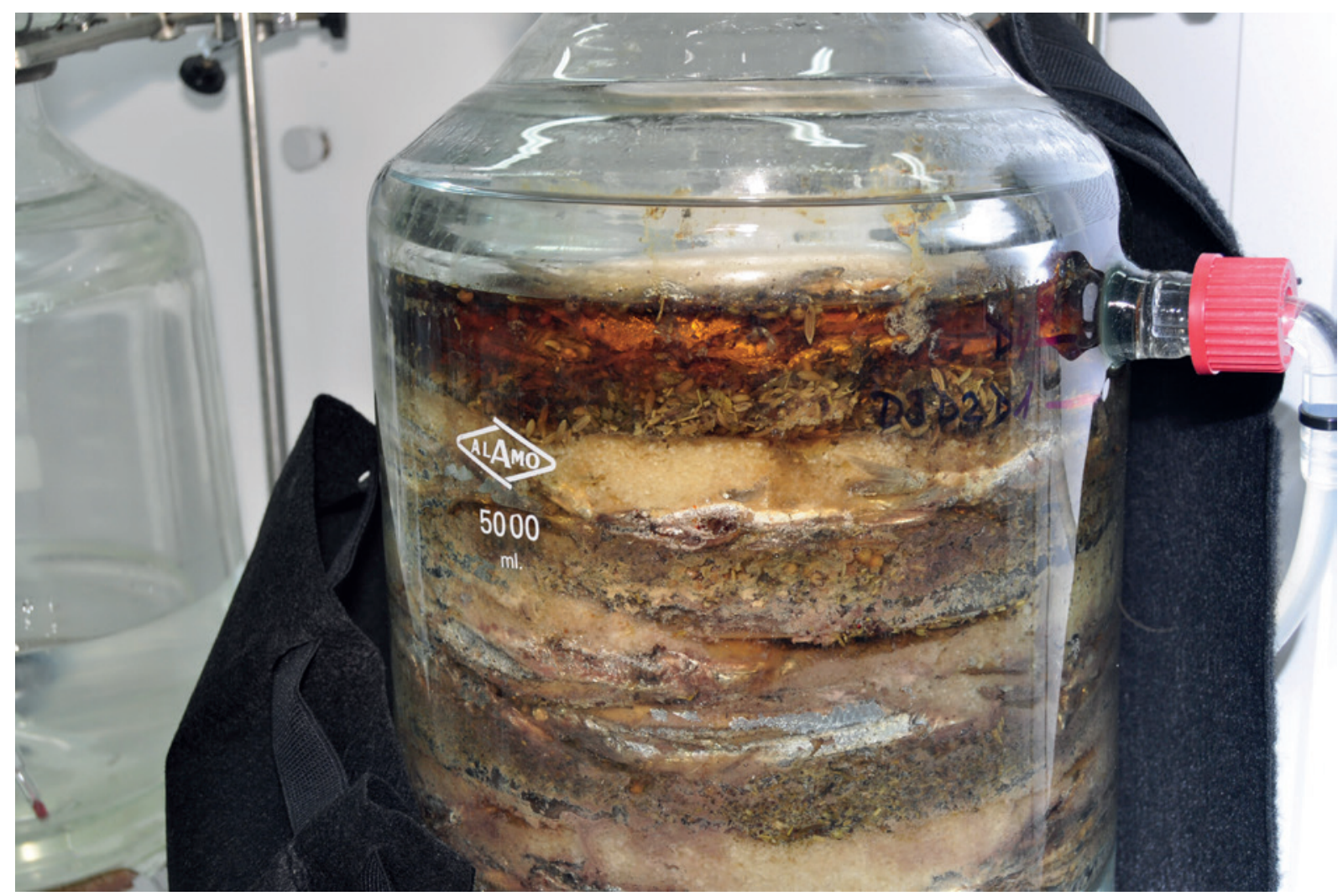

Figura 4. Fermentador con el producto durante el proceso de elaboración en el Laboratorio de Tecnología de los Alimentos de la Universidad de Cádiz.

sal; entonces, se cierra poniéndole un tapón y se deja siete días, transcurridos los cuales, se mueve hasta el fondo la preparación dos o tres veces al día durante veinte con un palo de madera en forma de remo. Hecho lo cual, se recoge el líquido que fluyó (defluxit) de esta preparación.

Y de este modo se hace el liquamen u omogarum a partir de lo anterior: se toman dos sextarios de este licor y se mezclan con medio sextario de buen vino, después se echa en esta mezcla un manojo de cada una de cuatro hierbas aromáticas (...). Todas ellas reducidas a pedazos muy pequeños se añaden al licor. Después, en un recipiente de hierro o de bronce se cuece la mezcla el tiempo necesario para que se reduzca el volumen de un sextario. Conviene, sin embargo, añadir a ella antes de la cocción media libra de miel espumada. Cuando estuviese cocida, debes colarla con un saco como los ungüentos hasta que claree y debe ser introducida hirviendo en el saco. Una vez clarificada y refrigerada, se conserva como condimento en un recipiente bien empegado.»

(Traducción E. García Vargas).

\section{MATERIALES Y MÉTODOS}

\subsection{Montaje experimental de la receta}

Una vez obtenidos los ingredientes, nos dispusimos a elaborar garum o liquamen, en recipientes que se acercaran lo más posible al diseño de los dolia en los que creíamos se había fabricado en la Bottega del Garum de Pompeya, y en las condiciones de elaboración lo más próximas posible a un entorno "natural". Se usó un fermentador dotado de una camisa de refrigeración con agua y conectado a un baño termostatizado para mantener la temperatura constante durante todo el experimento (fig. 4). La temperatura elegida es la que se espera que se pudiera alcanzar en verano a pleno sol en el interior del dolium; con sus variaciones durante el día y la noche. Los ingredientes se fueron añadiendo por separado, las especias en primer lugar, sobre ellas, unos 500-700 gramos de pescado, cubierto por 250-300 gramos de sal. Todo ello constituía una capa que se fue repitiendo hasta llenar todo el 
recipiente con 3,5 kilogramos de pescado, 1,5 kilogramos de sal y especias. Una vez transcurrido el tiempo que reseña Marcial en su receta, y habiendo realizado los procesos mecánicos que en ella se señalan, se filtró el líquido resultante a través de una tela de lino. Primero se dejó filtrar por simple gravedad, recogiendo un primer liquamen y tras presionar la pasta de pescado sobre la tela, se recogió una segunda porción considerada garum o liquamen secundum. La mezcla de pescado sal y especias, que, según las descripciones antiguas, constituiría el hallec, se dejó secar dentro del lino. Todos los productos obtenidos se guardaron refrigerados o congelados para su posterior análisis físicoquímico y sensorial, excepto la salmuera que se formó sobre las capas alternas de pescado, sal y especias en los días anteriores al removido del producto y que debe constituir el preparado que las fuentes literarias denominan muria. En nuestro caso, esta salmuera se mezcló con el resto de la composición al mezclar los ingredientes removiéndolos durante los veinte días posteriores a la semana de maceración, pero es muy probable que en los saladeros antiguos se extrajese de alguna forma de las salsamenta antes de proceder a remover el producto para la obtención del garum y el hallec. En futuras fases de nuestro trabajo, consideraremos la posibilidad de separar esta muria inicial, sin duda el más barato y fácil de obtener de los subproductos del liquamen, con idea de caracterizarla bioquímicamente.

\subsection{Análisis bioquímico de las muestras de Pompeya}

Para el segundo de los procedimientos técnicos se ha realizado una caracterización bioquímica de los restos piscícolas encontrados en seis dolia o grandes contenedores conservados en el Ambiente 9 de la Bottega del Garum de Pompeya (I, 12, 8). Como patrón de referencia se han empleado, para algunas analíticas, muestras actuales de restos óseos de anchoa de la misma especie caracterizada en las muestras arqueológicas. A continuación se describe el material, el instrumental y la metodología empleada en los diferentes análisis.

\subsubsection{Estudio macroscópico}

Pevio al análisis químico se realizó un estudio macroscópico de las muestras para caracterizar la naturaleza de los sólidos y fragmentos con objeto de facilitar la interpretación de los resultados. El estudio macroscópico se realizó con una lupa binocular LEICA
ZOOM 2000 con capacidad para 10x y 40x aumentos, acoplada a una cámara digital. En el estudio se analizó: color (tonalidad), tamaño de los fragmentos y homogeneidad de la muestra (fig. 7).

\subsubsection{Contenido en materia orgánica, cenizas y composición mineral}

Para la determinación del porcentaje de materia orgánica y cenizas, las muestras se calcinaron a $550^{\circ} \mathrm{C}$ en un horno MUFLA, según el método descrito por la AOAC (1990). A continuación, tras homogeneizar, se realizó una digestión ácida, tras lo cual se analizó la composición mineral (P, Ca, Na, Mg, K, Fe, Cu y Zn) mediante espectrofotometría de absorción atómica en la modalidad de plasma (ICP-AES) con un espectrógrafo ICP-MS THERMO/SERIE X7.

\subsubsection{Contenido en grasas}

El contenido en grasas se determinó mediante gravimetría por el método Soxhlet recogido por Suzanne (2007). Se empleó un equipo de extracción Sohxlet VELP SCIENTIFICA modelo SER 148. Las muestras se pesaron en cartuchos de celulosa y fueron sumergidas en hexano a $130^{\circ} \mathrm{C}$ durante 60 minutos. Posteriormente se lavaron durante 60 minutos, seguidos de 30 minutos de recuperación del disolvente. El contenido en grasas se obtiene de la diferencia de peso de la muestra antes y después de la extracción.

\subsubsection{Análisis de ácidos grasos}

El contenido en ácidos grasos se determinó por cromatografía de gases, según el método descrito por Gómez (2000). Se empleó un cromatógrafo de gases Hewlett Packard 5890 GC con detector de ionización FID, y una columna de fase Teknokroma TBR-WAX. Los valores de referencia se obtuvieron de un patrón estándar de ácidos grasos de productos marinos FAME, de la casa comercial RESTEK.

\subsubsection{Análisis de aminoácidos}

Para obtener el perfil de aminoácidos, se tomaron 10 gramos de muestra de cada dolium y se prepararon para su derivatización con OPA, previo a su análisis por 
cromatografía líquida de alta resolución en un equipo WILSON dotado con columna SUPELCOSILTM LC, siguiendo la metodología de Betancort (1997).

\section{RESULTADOS Y DISCUSIÓN}

\subsection{Primer procedimiento técnico: obtención del producto a partir de la receta}

El liquamen obtenido a partir de la receta establecida en el apartado 3.1. resultó ser un líquido de color pardo-ocre con aspecto aceitoso y saturado de sal, con olor suave a pescado y especias (fig. 5). El allec, de aspecto bastante homogéneo y pastoso, desprende un fuerte olor a especias y pescado en salazón, su sabor es menos salado que el liquamen y conserva sabor a pescado y especias, con predominio de las lamiáceas más aromáticas (fig. 6). Se diferencia del liquamen también en su aspecto físico, pues conserva toda la fracción ósea de los animales no filtrada y convertida en pequeñísimos fragmentos con escasas aristas y ninguna espina completa, debido al alto grado de micronización, provocado por el removido continuo durante semanas de la mezcla, que fragmenta y redondea los huesos de los peces hasta el punto de que es posible consumir la pasta (hallec) sin problemas derivados de la presencia de espinas o grandes fragmentos del esqueleto de los animales. Este es uno de los resultados más inesperados del proceso: la posibilidad de identificar el hallec a partir del estado físico de los residuos, desechando como tal salsa los restos óseos a menudo identificados

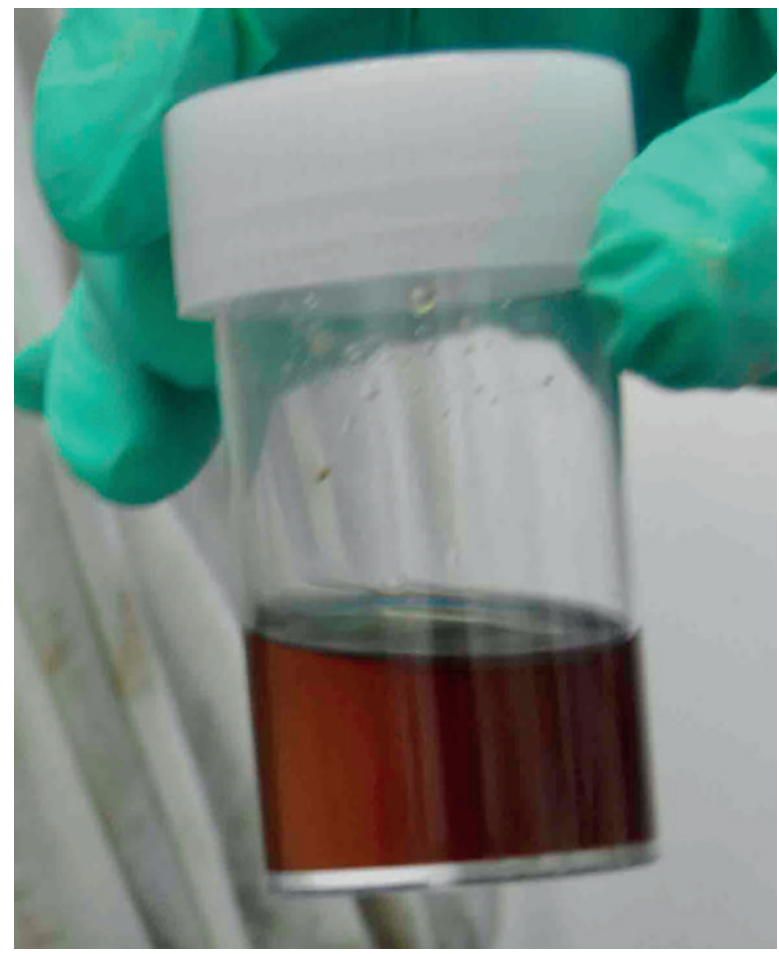

Figura 5. Producto líquido o garum/liquamen obtenido.

como tal por no hallarse en conexión anatómica pero que conservan su tamaño y forma originales; para que un sedimento se identifique con hallec debe estar constituido por restos óseos micronizados (vide infra apartado 4.2.1. Resultado del estudio macroscópico).
Figura 6. Detalle del producto sólido o hallec obtenido.

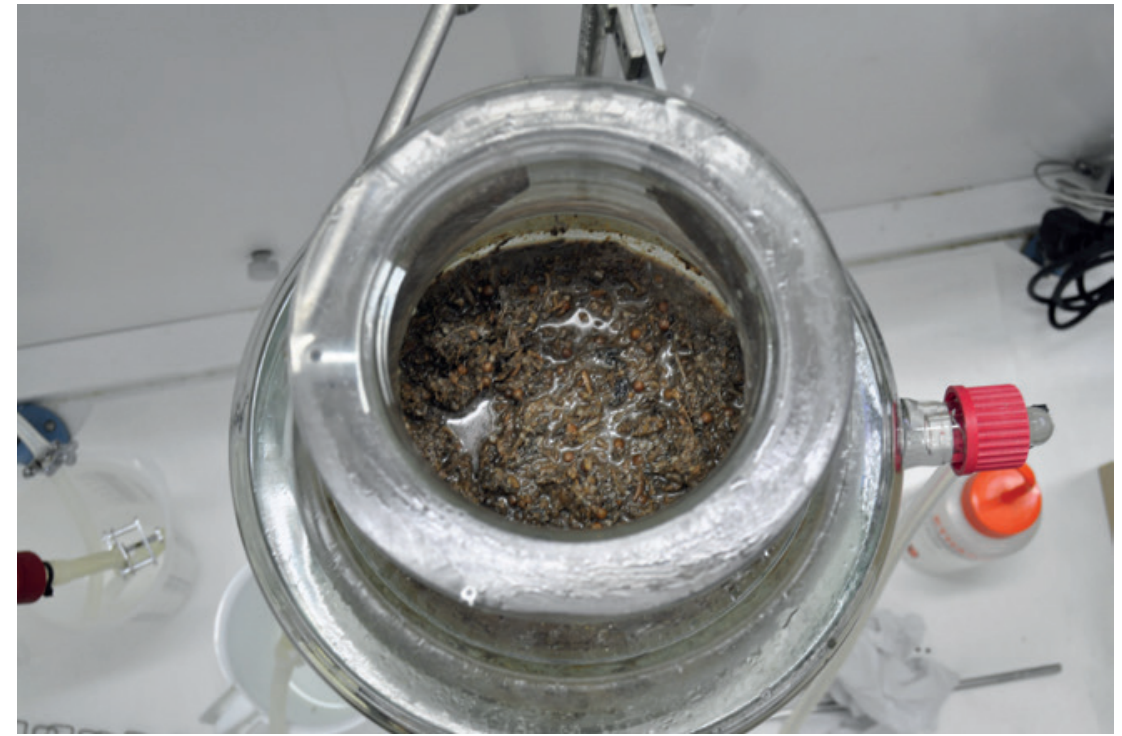



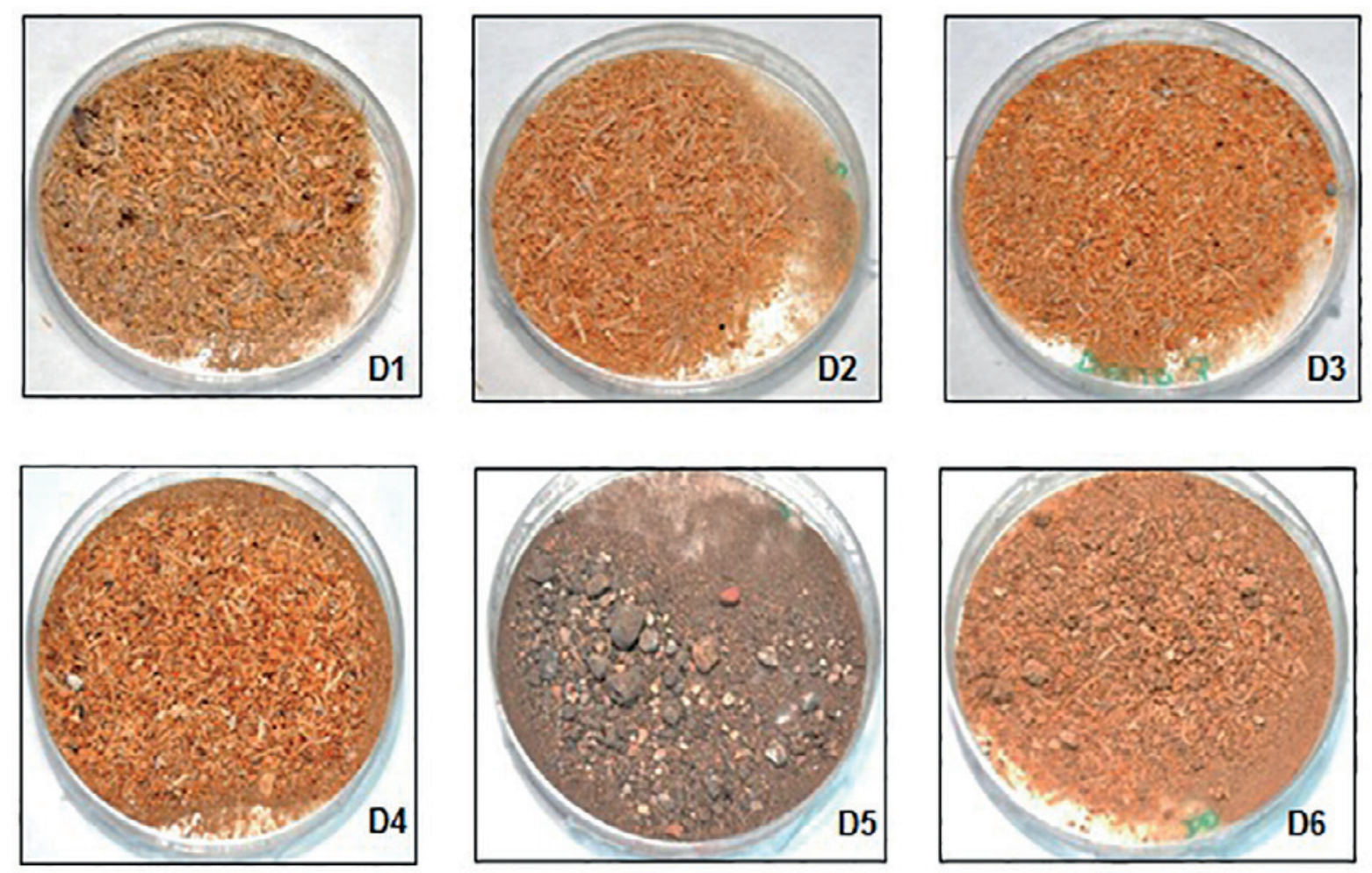

Figura 7. Detalle de las muestras analizadas de los dolia de Pompeya.

\subsection{Segundo procedimiento técnico: análisis bioquímico de la muestra arqueológica pompeyana y comparación con los productos obtenidos en laboratorio}

Los trabajos de análisis arqueofaunístico y bioquímico en restos de salazones y salsas de pescado documentados en contenedores arqueológicos tienen ya una relativamente larga tradición en Arqueología (recientemente, Van Neer, Ervynck y Monsieur 2010). Sin embargo, la mayoría de ellos está dedicada a la determinación de las especies ícticas presentes en la muestra, siendo escasa y circunstancial la atención prestada a la determinación del tipo de salazón de pescado contenida originalmente en los recipientes analizados. Las conclusiones, cuando las hay, al respecto de esta última cuestión derivan habitualmente de observaciones simples sobre la disposición física de los restos óseos analizados. Suele considerarse que los restos de peces en conexión anatómica corresponden a productos sólidos de pescado (salsamenta), mientas que aquellos que carecen de continuidad anatómica serían más bien el resultado de la mezcla de sus componentes durante procesos más o menos complejos de obtención de salsas saladas de pescado. De entre éstas últimas, y sobre la base de la afirmación (supra) de Plinio (imperfecta nec colata faex), suelen atribuirse al hallec los compuestos que muestran un número elevado de espinas y vértebras inconexas en la composición del residuo, mientras que la presencia de partes específicas de la anatomía de los peces, como las piezas óseas faringobranquiales, denunciaría un producto tipo garum confeccionado con los aparatos branquiales, las agallas, la sangre y el suero de los peces (Van Neer y Parker 2008).

En los últimos años, se ha unido al estudio de los restos óseos el de los ácidos grasos presentes en las muestras analizadas como medio de aproximación a la composición bioquímica del producto originalmente envasado en los contenedores estudiados, y se está trabajando sobre la potencial presencia de proteínas (Dallongeville et alii 2010; así como en varios trabajos en las Actas del I Congreso Internacional de la SECAHEx officina hispana). Un trabajo reciente (Smriga et alii 2010) compara el contenido en aminoácidos libres de las salsas de pescado actuales de Italia y Asia con los de las salsas romanas (cf. Lowe 2009) o mejor, con los 
restos de aminoácidos (esp. glutamato) documentados por la analítica bioquímica realizada a los restos de sedimento contenido en los dolia de la Bottega del Garum (I, 12, 8) de Pompeya, el mismo residuo del que procede la muestra sobre la que hemos trabajado nosotros en laboratorio. Otra analítica reciente (Ribechini et alii 2009) concluye, tras una multianalítica química, que la presencia de cristales de sal, espinas de peces y lípidos animales en una jarra pintada tardía (siglo VII d.C.) de Antinoe (Egipto), en la que se detectan igualmente trazas orgánicas de resina de pino, corresponde a un contenido original consistente con una salsa de pescado de tipo garum, allec, muria o liquamen.

Aunque se trata de trabajos pioneros y de indudable interés como referencia metodológica y analítica, siempre queda la impresión de que el análisis puede llevarse más adelante hasta poder diferenciar, o tratar de hacerlo, calidades o tipos concretos de producto y no sólo preparaciones genéricas tipo "salsa". Esta es la intención de nuestro segundo procedimiento técnico: la determinación de la composición de la muestra de referencia mediante el estudio macroscópico y la analítica química.

En concreto, se trata de obtener, mediante reproducción de los procesos técnicos implicados en su fabricación, un producto lo más parecido posible a las salsas originales romanas y comparar su aspecto, cualidades físico-químicas, textura y composición con la observada en muestras extraídas de contextos arqueológicos concretos. Sobre el papel, es la manera más fiable de determinar no solo la naturaleza concreta de cada producto (garum, hallec, muria, liquamen), sino, sobre todo, de reconocer la clase de residuo que la producción de cada una de estas salsas, o al menos de algunas de ellas, genera en el registro arqueológico.

\subsubsection{Resultados del estudio macroscópico}

En todos los dolia, salvo en el dolium 5 se encuentran restos óseos constituidos por vértebras y espinas de pescado que según Curtis (1979) corresponden a la especie Engraulis encrasicolus (L 1758), el conocido boquerón mediterráneo, y que analizados recientemente por miembros del equipo (R. Marlasca y C.G. Rodríguez Santana), han aportado datos similares, además de detectar la existencia puntual de otras especies piscícolas minoritarias (fig. 8). Actualmente estos datos están en fase de publicación, habiéndose únicamente dado a conocer algunos de los avances del trabajo de campo (Bernal, Cottica y Zaccaria 2009; Bernal et alii 2013). Algunas de las vértebras que aparecen enteras en los distintos dolia son de

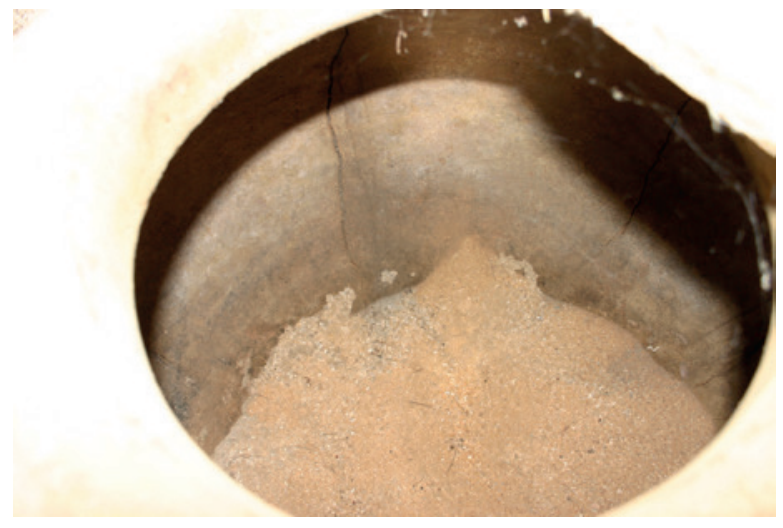

Figura 8. Ictiofauna conservada en el interior del dolium $\mathrm{n}^{\circ} 3$ de la "tienda del garum" de Pompeya.

tamaño muy similar, lo que indica que se trata de ejemplares de misma especie y talla, tal como había sido constatado por Curtis (1979) y Sternberg (2007).

El nivel de micronizado se detalla en la tabla 1. Se ha podido comprobar que el dolium $\mathrm{n}^{\mathrm{o}} 1$ contiene la mayor cantidad de fragmentos enteros, seguido del $d o-$ lium $\mathrm{n}^{\mathrm{o}} 2$, encontrándose en los dolia $\mathrm{n}^{\mathrm{os}} 3,4$ y 6 los fragmentos más pequeños, muy micronizados, incluso en tamaño polvo. Los dolia $\mathrm{n}^{\text {os }} 3$ y 4 contienen mayores cantidades de polvo (de origen animal, vegetal o mineral), siendo posible observar en el dolium $\mathrm{n}^{\circ} 4$ una tonalidad más oscura, muy probablemente por tener mayor cantidad de micronizado de color negro, posiblemente de origen volcánico. El dolium $\mathrm{n}^{\mathrm{o}} 6$ presenta gran cantidad de fragmentos de roca y polvo de diferentes tonalidades y tamaños que el resto, por lo que pudo estar más expuesto al ambiente que los otros.

Tabla 1. Valoración del micronizado de la fracción ósea y el resto de la muestra.

\begin{tabular}{|c|c|c|c|c|c|c|}
\cline { 2 - 7 } \multicolumn{1}{c|}{} & $\begin{array}{c}\text { Dol. } \\
\mathrm{n}^{\mathrm{o}} 1\end{array}$ & $\begin{array}{c}\text { Dol. } \\
\mathrm{n}^{\circ} 2\end{array}$ & $\begin{array}{c}\text { Dol. } \\
\mathrm{n}^{\circ} 3\end{array}$ & $\begin{array}{c}\text { Dol. } \\
\mathrm{n}^{\mathrm{o}} 4\end{array}$ & $\begin{array}{c}\text { Dol. } \\
\mathrm{n}^{\circ} 5\end{array}$ & $\begin{array}{c}\text { Dol. } \\
\mathrm{n}^{\circ} 6\end{array}$ \\
\hline $\begin{array}{c}\text { Fracción } \\
\text { Ósea }\end{array}$ & $3 *$ & 2 & 1 & 1 & $\varnothing$ & 1 \\
\hline $\begin{array}{c}\text { Resto de } \\
\text { muestra }\end{array}$ & 1 & 1 & 1 & 1 & 3 & 2 \\
\hline
\end{tabular}

* Se otorgó un valor numérico al grado de micronización, siendo el 3 el mayor valor y 1 el menor. $\varnothing$ señala ausencia de fragmentos óseos visibles.

Como se señala anteriormente, el dolium $\mathrm{n}^{\circ} 5$ no revela a simple vista restos óseos de pescado y presenta una tonalidad muy oscura, con posibles intrusiones de minerales (fig. 7). 


\subsubsection{Contenido en materia orgánica y cenizas}

En la tabla 2 se recogen los resultados correspondientes al porcentaje de materia orgánica y de cenizas de los dolia. Los niveles de materia orgánica observados oscilan entre un 10 y un $15 \%$, salvo en el dolium 5, cuya fracción orgánica es tan solo del 3,75\%. Estos resultados nos indican que aún se conservan restos orgánicos del producto que contuvieron originalmente y que proceden de los fragmentos óseos (espinas, vértebras, etc.) y de algunas posibles adherencias que hayan quedado sobre ellos (tab. 2). No obstante los niveles de materia inorgánica (cenizas) encontrados son muy elevados, lo que indica el nivel de degradación sufrido por el o los productos a lo largo del tiempo. Por otra parte, los niveles bajos de materia orgánica encontrados en el dolium 5 se pueden justificar por la escasa presencia de restos óseos y su riqueza en materia inorgánica (cenizas), cercana al $97 \%$. Esto hace pensar en la hipótesis de que el dolium 5 contenía un producto de naturaleza y/o elaboración distinta, o bien que había estado más expuesto al ambiente desde la erupción que el resto de dolia, aunque no hay indicios arqueológicos que induzcan a pensar en esta última posibilidad.

Tabla 2. Contenido en materia orgánica e inorgánica en los restos de los dolia.

\begin{tabular}{|c|c|c|}
\hline Muestra & $\begin{array}{c}\text { Materia } \\
\text { Orgánica (\%) }\end{array}$ & $\begin{array}{c}\text { Materia } \\
\text { Inorgánica (\%) }\end{array}$ \\
\hline Dol. $\mathrm{n}^{\circ} 1$ & 15,37 & 84,63 \\
\hline Dol. $\mathrm{n}^{\circ} 2$ & 12,77 & 87,23 \\
\hline Dol. $\mathrm{n}^{\circ} 3$ & 9,86 & 90,14 \\
\hline Dol. $\mathrm{n}^{\circ} 4$ & 12,96 & 87,04 \\
\hline Dol. $\mathrm{n}^{\circ} 5$ & 3,75 & 96,25 \\
\hline Dol. $\mathrm{n}^{\circ} 6$ & 11,64 & 88,36 \\
\hline
\end{tabular}

\subsubsection{Contenido en grasas}

Como puede observarse en la tabla 3, los contenidos en grasas, expresados en porcentaje de peso seco, oscilan entre 1.5 y $5 \%$ aproximadamente, salvo el $d o$ lium $\mathrm{n}^{\circ} 5$ que presenta el porcentaje más bajo $(0,67 \%)$. Atendiendo al porcentaje de grasas podemos establecer dos agrupaciones: un primer grupo, constituido por los dolia $\mathrm{n}^{\text {os }} 1,2$ y 6 , con niveles inferiores al $2 \%$ y un segundo grupo formado por los dolia $\mathrm{n}^{\mathrm{os}} 3$ y 4 , cuyo porcentaje de grasas se sitúa por encima del $4 \%$. No existe una relación entre el contenido en materia orgánica y el contenido en grasas de los dolia, por lo que parece obvio que gran parte de esta materia orgánica esté constituida por compuestos nitrogenados de diferente origen.

Tabla 3. Contenido en grasas de los dolia.

\begin{tabular}{|c|c|}
\hline Muestra & Contenido en grasas (\% peso seco) \\
\hline Dolio 1 & 1,63 \\
\hline Dolio 2 & 1,60 \\
\hline Dolio 3 & 4,15 \\
\hline Dolio 4 & 4,59 \\
\hline Dolio 5 & 0,67 \\
\hline Dolio 6 & 1,29 \\
\hline
\end{tabular}

Las grasas presentes en los restos de los dolia proceden fundamentalmente de los fragmentos óseos, y en menor medida, de las especias usadas como saborizantes y aromatizantes en el proceso.

\subsubsection{Contenido en ácidos grasos.}

Para corroborar que la fracción grasa encontrada en los dolia procede de fragmentos óseos, se estudió la composición de los ácidos grasos, incluyendo una muestra de referencia de restos óseos de anchoa. Como podemos observar en la tabla 4, el perfil de ácidos grasos de los dolia (incluyendo el $\mathrm{n}^{\circ}$ 5) es muy similar al de la anchoa. Estos resultados indican, por un lado que la fracción grasa y más concretamente los ácidos grasos de las muestras proceden fundamentalmente de los restos óseos de las anchoas presentes en el producto original, y por otro, que efectivamente en el dolium $\mathrm{n}^{\mathrm{o}} 5$ existen evidencias de restos óseos de producto en pequeña proporción, que no se pueden apreciar a nivel macroscópico por su nivel de micronización (tabla 3),

El ácido graso mayoritario que aparece en todas las muestras (dolia y anchoa) es el ácido miristoleico (28$67 \%$ ), seguido del ácido eicosanoico y del eicosapentanoico. Por su gran estabilidad, estos ácidos podrían servir de marcadores de procedencia de garum y derivados, en adherencias y restos arqueológicos para futuros trabajos. 
Tabla 4. Composición en ácidos grasos (\%) de los restos piscícolas de los dolia y la referencia.

\begin{tabular}{|c|c|c|c|c|c|c|c|}
\hline Ácido graso & Anchoa (óseo) & Dolio $\mathrm{n}^{\circ} 1$ & Dolio $n^{\circ} 2$ & Dolio $n^{\circ} 3$ & Dolio $n^{\circ} 4$ & Dolio $\mathrm{n}^{\circ} 5$ & Dolio ${ }^{\circ} 6$ \\
\hline Mirístico & 0,38 & 1,05 & 6,22 & 3,54 & 2,29 & 2,16 & 0,85 \\
\hline Miristoleico & 40,78 & 53,22 & 42,95 & 28,13 & 62,42 & 41,77 & 58,47 \\
\hline Palmítico & 0,93 & 0,00 & 0,00 & 0,00 & 0,00 & 2,83 & 1,86 \\
\hline Palmitoleico & 0,29 & 0,48 & 2,22 & 0,71 & 2,39 & 1,74 & 1,02 \\
\hline Heptadecanoico & 0,07 & 0,00 & 3,72 & 0,00 & 0,00 & 0,00 & 0,00 \\
\hline Estearico & 1,69 & 3,21 & 0,00 & 0,00 & 0,00 & 0,00 & 0,65 \\
\hline Oleico & 2,90 & 2,41 & 0,00 & 0,00 & 0,00 & 6,37 & 2,04 \\
\hline Linoleico & 0,00 & 0,00 & 0,00 & 27,04 & 0,00 & 1,62 & 0,67 \\
\hline Linolénico & 0,43 & 0,00 & 4,20 & 0,00 & 5,19 & 2,85 & 0,66 \\
\hline Eicosanoico & 15,93 & 18,58 & 13,08 & 26,64 & 15,58 & 13,31 & 27,19 \\
\hline Eicosadienoico & 7,98 & 6,82 & 16,02 & 4,16 & 4,25 & 6,26 & 6,01 \\
\hline Eicosatrienoico & 11,90 & 0,90 & 0,00 & 0,75 & 0,00 & 3,86 & 0,59 \\
\hline Eicosapentanoico & 16,72 & 13,31 & 11,58 & 9,02 & 7,88 & 17,22 & 0,00 \\
\hline
\end{tabular}

\subsubsection{Composición en aminoácidos}

Los resultados correspondientes al aminograma de los dolios se presentan en la tabla 5 . Como se puede observar para todas las muestras, los valores de aminoácidos se encuentran por debajo del límite de detección del equipo, por lo que se hace necesario el empleo de un equipo de cromatografía líquida acoplado a un espectrógrafo de masas, para poder cuantificar los aminoácidos en unidades de $\mu \mathrm{g} / 100 \mathrm{mg}$.

\subsubsection{Composición mineral}

En cuanto a la composición mineral, se observa que las proporciones en los dolia no son tan similares entre sí como en el caso de los ácidos grasos. Sin embargo, sí se observan en todos los dolia (salvo en el 5) y en muestra referencia (fracción ósea de la anchoa), que los compuestos mayoritarios son el Ca y el P (tabla 6). El tejido óseo está compuesto mayoritariamente por una matriz extracelular de naturaleza orgánica, cubierta por hidroxiapatito $\left[\mathrm{Ca}_{5}\left(\mathrm{Po}_{3}\right)_{3} \mathrm{OH}_{2}\right]$ (Toppe et alii 2007). Por esta misma razón, debe existir una relación entre los contenidos en Ca y P para todos los dolia. Tras realizar un análisis de correlación entre ambos elementos se ha
Tabla 5. Aminograma de los restos encontrados en los dolia.

\begin{tabular}{|c|c|}
\hline $\begin{array}{c}\text { Aminograma } \\
\text { (D1, D2, D3, D4, D5 y D6) }\end{array}$ & Resultado (\%) \\
\hline Valina & $<0.01$ \\
\hline Treonina & $<0.02$ \\
\hline Tirosina & $<0.01$ \\
\hline Serina & $<0.01$ \\
\hline Metionina & $<0.01$ \\
\hline Lisina & $<0.01$ \\
\hline Leucina & $<0.01$ \\
\hline Isoleucina & $<0.01$ \\
\hline Histidina & $<0.01$ \\
\hline Glicina & $<0.01$ \\
\hline Fenilalanina & $<0.01$ \\
\hline Arginina & $<0.01$ \\
\hline Alanina & $<0.01$ \\
\hline Ácido Glutámico & $<0.01$ \\
\hline Ácido Aspártico & $<0.01$ \\
\hline & \\
\hline
\end{tabular}


Tabla 6. Composición mineral de los dolia y la referencia (mg de mineral en 100 gramos de muestra).

\begin{tabular}{|c|c|c|c|c|c|c|c|}
\hline Elemento & Dol. $n^{\circ} 1$ & Dol. $n^{\circ} 2$ & Dol. $n^{\circ} 3$ & Dol. $n^{\circ} 4$ & Dol. $n^{\circ} 5$ & Dol. $n^{\circ} 6$ & Anchoa \\
\hline $\mathrm{P}$ & 9115,00 & 13539,60 & 14660,00 & 13257,43 & 504,95 & 12524,75 & 1566,83 \\
\hline $\mathrm{Ca}$ & 19770,00 & 27242,57 & 28525,00 & 25128,71 & 22891,09 & 25688,12 & 2829,21 \\
\hline $\mathrm{Na}$ & 545,00 & 466,83 & 356,00 & 400,99 & 159,41 & 284,16 & 158,66 \\
\hline $\mathrm{Mg}$ & 565,00 & 673,27 & 170,00 & 277,23 & 208,42 & 366,34 & 60,64 \\
\hline K & 277,50 & 83,17 & 90,50 & 99,50 & 544,55 & 63,37 & 398,51 \\
\hline $\mathrm{Fe}$ & 386,50 & 368,81 & 353,00 & 425,74 & 712,87 & 337,62 & 3,09 \\
\hline $\mathrm{Cu}$ & 4,85 & 5,45 & 4,99 & 6,14 & 4,42 & 3,89 & 0,50 \\
\hline $\mathrm{Zn}$ & 40,65 & 93,07 & 86,50 & 69,31 & 5,45 & 69,31 & 3,96 \\
\hline Prop. $\mathrm{Ca} / \mathrm{P}$ & 2,17 & 2,01 & 1,95 & 1,90 & 45,33 & 2,05 & 1,81 \\
\hline
\end{tabular}

verificado un coeficiente de correlación $\mathrm{R}^{2}$ de 0,99 , si excluimos el dolium $\mathrm{n}^{\circ} 5$ del análisis. Así mismo, en la tabla 6 se expone la relación entre los valores de Ca y $\mathrm{P}$ para las distintas muestras. Como se puede apreciar, los valores $\mathrm{Ca} / \mathrm{P}$ son muy parecidos en los dolia $\mathrm{n}^{\text {os }} 1,2$, 3,4 y 6 (promedio 2,01) y muy cercanos al valor de la

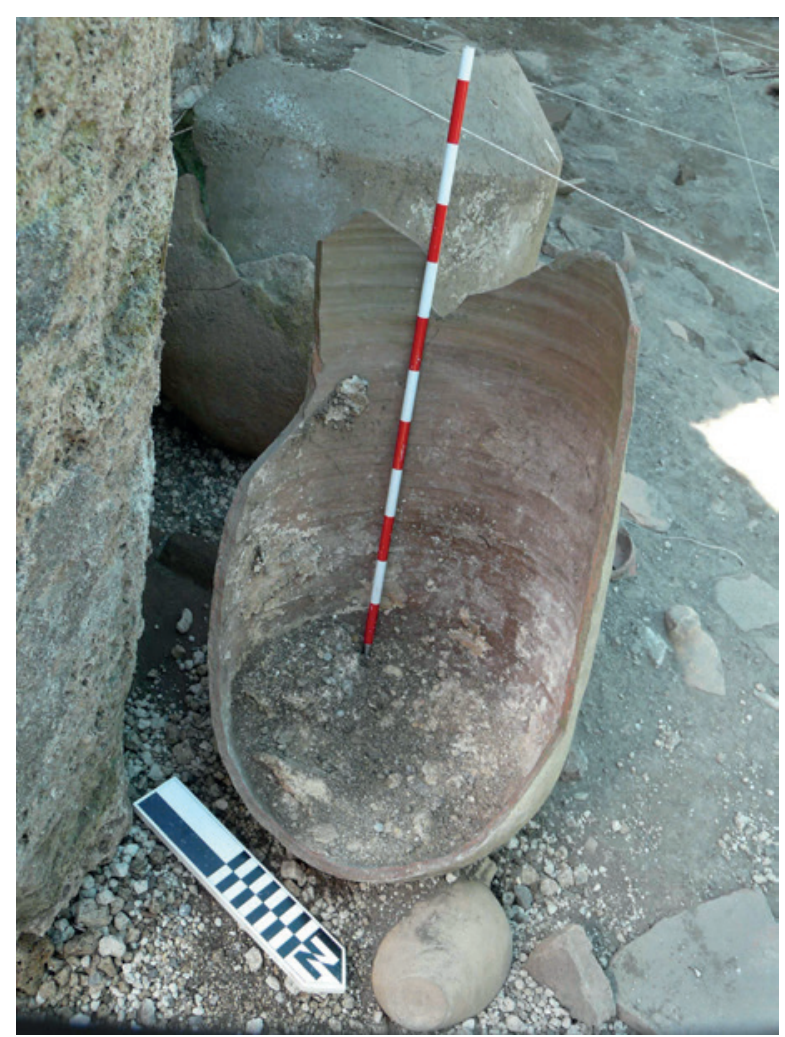

anchoa (1,81). Por tanto, a la vista de estos resultados, podemos asegurar que la fracción mineral, correspondiente a $\mathrm{Ca}$ y $\mathrm{P}$ procede, fundamentalmente, del tejido óseo de pescado en estos dolia.

En el dolium $\mathrm{n}^{\circ} 5$ los niveles de $\mathrm{P}$ encontrados vuelven a constatar, como en el apartado de ácidos grasos, la presencia de restos óseos en pequeña proporción. Sin embargo, los niveles de Ca encontrados son muy superiores a los esperados, según la proporción de $\mathrm{P}$, lo que hace que la relación $\mathrm{Ca} / \mathrm{P}$ en el dolium $\mathrm{n}^{\circ} 5$ sea muy superior al del resto de dolia (45,33 frente a 2,01). Esto parece indicar que el Ca que aparece en el dolium $\mathrm{n}^{\circ} 5$ no proviene solo de los restos óseos, sino que mayoritariamente procede de la adición o incorporación de cal posiblemente durante el proceso de elaboración, como agente neutralizante de la acidez. Hay evidencias de la presencia de un ánfora que contenía un producto calcáreo dentro del ambiente 13 -anexo al ambiente 9(fig. 9). Tras un análisis de difracción de rayos $\mathrm{X}$ se ha comprobado que su composición es calcita, o sea carbonato cálcico, lo que viene a indicar que en su origen pudo ser cal viva.

Sabemos por estudio de salazones de pescado realizados en laboratorio (datos no publicados) que el $\mathrm{pH}$ del tejido muscular disminuye significativamente hasta

Figura 9. Proceso de excavación del saggio 4 del ambiente 13, con un ánfora africana reutilizada, con restos de cal en su interior. 
valores de $\mathrm{pH}$ en torno a 5 , sobre todo en el caso de salazón de anchoa. Dicha reducción de $\mathrm{pH}$ puede ser más acusada en el caso de operar con pescado muy fresco. Los $\mathrm{pH}$ bajos producen una inhibición drástica de la actividad proteasa, fenómeno que ocurre tanto en músculo, como en víscera; generando menos liquamen y con mayor acidez. Por otra parte, se puede observar que el Fe se encuentra en una proporción más elevada en los dolia respecto a la muestra de referencia ósea de la anchoa. Estos resultados pueden deberse a diversas razones: a) la presencia de mayores cantidades de sangre y vísceras en los dolia, b) las intrusiones que se han ido produciendo desde el momento de la erupción (piedra pómez o volcánica), y c) el aporte mineral del resto de los posibles ingredientes del allec, entre otras hipótesis. Los niveles altos de $\mathrm{K}$ de la muestra de anchoa se explican por la aportación que hacen los restos musculares que quedan adheridos a la espina. Las bajas proporciones de $\mathrm{Na}$ y $\mathrm{K}$ en los dolia pueden deberse al efecto de la lixiviación, dada la alta solubilidad de estos minerales.

\section{CONCLUSIONES}

El trabajo realizado en colaboración entre las universidades de Sevilla y Cádiz ha permitido reproducir en condiciones controladas de laboratorio los procesos de elaboración artesanal de tres salsas de pescado romanas citadas por las fuentes clásicas y cuyos nombres comparecen igualmente en los rótulos pintados de las ánforas salsarias: el liquamen, el hallec y la muria, así como su ulterior comparación con los residuos obtenidos de los dolia de la bottega del garum $(\mathrm{I}, 12,8)$ de Pompeya.

Consideramos, en este contexto, que el liquamen es un producto muy similar al garum, con el que en época tardía se acaba confundiendo, pero del que en momentos republicanos y altoimperiales se diferenciaba por proceder de peces de pequeño tamaño que se utilizaban completos, incluido el tracto digestivo, responsable de los procesos de hidrólisis que licuaban la carne de los peces. Éstos, junto a la sal y a las hierbas que cumplían funciones aromáticas y antisépticas, eran el ingrediente principal del producto. El compuesto obtenido por nosotros en laboratorio es una salsa muy líquida y de color ámbar en la que el sabor salado predomina sobre el sabor a pescado y en la que el fondo aromático proporcionado por las hierbas la aleja de cualquier consideración de producto maloliente o pútrido que, por razones morales, suele tener en determinados autores antiguos
(Curtis 1983). Somos conscientes de que el producto básico obtenido por nosotros es susceptible de una elaboración posterior, como señala la receta de Gargilio Marcial que hemos tomado como base, y que esta elaboración ulterior reduciría el volumen de producto a un tercio aproximadamente mediante la cocción del mismo, espesándolo seguramente y dándole una consistencia más pastosa, además de añadirle nuevos matices aromáticos por la adición de más hierbas, vino y miel. Este proceso de cocción, que sustituye a la exposición prolongada al sol de hasta tres meses que se señala en los Geoponica, permitiría una obtención más rápida de la pasta y aparece en todas las recetas que se conservan hasta hoy de la confectio gari. Nosotros hemos obtenido un liquamen base similar al que se debió fabricar en los dolia de la Bottega del garum de Pompeya de la que procede el residuo analizado también por nosotros y que no presentan indicios de combustión, aunque sí de adición de cal viva para rebajar el $\mathrm{pH}$ $\mathrm{y}$, eventualmente, generar calor.

El residuo del tamizado con una tela de lino o similar de la mezcla de pescado, sal y hierbas removida durante veinte días, es decir, el subproducto del liquamen (supra) es una pasta (hallec) de restos de carne imperfectamente hidrolizados, hierbas aromáticas y fragmentos del esqueleto óseo tremendamente micronizados, es decir, reducido a pequeños fragmentos sin puntas ni aristas, que se deja consumir como un condimento muy aromatizado de segunda calidad y, seguramente, menor coste.

Aún más barata debió ser la muria o salmuera que sobrenadaba el compuesto. No la hemos separado para no alterar el proceso de confección del liquamen, pero hemos observado su formación y aspecto, y queda pendiente de estudios futuros.

Una vez obtenidos los tres productos "históricos" se han comparado con los residuos extraídos de los dolia pompeyanos (fechados con precisión en agosto de 79 d.C.) caracterizados en su aspecto macroscópico, su contenido en materia orgánica, en ácidos grasos y en grasas, su composición en aminoácidos y su composición mineral, observándose que las diferencias de apariencia, composición y características bioquímicas de los mismos corresponden aproximadamente con las que se documentan en los productos elaborados en laboratorio. De este modo, puede proponerse que:

- Los dolia $\mathrm{n}^{\text {os }} 1$ y 2 que contienen fragmentos muy completos del esqueleto óseo de los peces corresponden a productos de pescado salado (¿aún?) no procesados para la obtención del $l i$ quamen. 
- Los dolia $\mathrm{n}^{\text {os }} 3,4$ y 6 que contienen partes del esqueleto muy micronizadas debieron contener en el momento de la erupción un producto de tipo hallec.

- El dolium $\mathrm{n}^{\circ} 5$ que no presenta restos orgánicos a simple vista, un mayor contenido en materia inorgánica como consecuencia de la erupción y un contenido graso claramente menor que el resto, pero, sin embargo, ofrece índices de ácidos grasos similares a los del resto de los dolia, bien contuvo un producto similar al de los dolia 3, 4 y 6 y estaba vacío en el momento de la erupción, o bien, y es lo más probable, contenía un producto líquido con fragmentos óseos indetectables al examen macroscópico, aunque presentes, ya que los ácidos grasos de las muestras analizadas proceden fundamentalmente de los restos óseos de las anchoas (¿liquamen completamente elaborado y filtrado?).

Las posibilidades son varias: o bien estamos ante un grupo de contenedores de los que la mayoría contenían un liquamen en diverso estado de elaboración y sólo uno $\left(\mathrm{el}^{\circ} 5\right)$ contenía producto ya elaborado, o bien nos encontramos ante dolia expuestos a la venta que incluían pescado salado no fermentado ( $\mathrm{n}^{\text {os }} 1$ y 2$)$, hallec $\left(\mathrm{n}^{\circ \mathrm{s}} 3,4\right.$ y 6$)$ y liquamen $\left(\mathrm{n}^{\circ} 5\right)$. En cualquier caso, las similitudes en composición físico-química y en aspecto físico entre los productos elaborados en laboratorio y los residuos de salsas de pescado de origen arqueológico abren una vía de investigación importante para la correcta adscripción de estos últimos, sobre la cual se sigue trabajando en la actualidad.

\section{Agradecimientos}

Este trabajo se inscribe en el marco de desarrollo de los proyectos de investigación 15733, HAR201128244 y HAR2013-43599 del Plan Nacional de I+D+i del Ministerio de Competitividad/FEDER del Gobierno de España. Las muestras analizadas se obtuvieron en el transcurso de las excavaciones en la Bottega del Garum de Pompeya, como parte de las tareas del proyecto internacional El Garum de Pompeya y Herculano. Explotación de los recursos del mar en el ámbito vesubiano. Este proyecto ha sido apoyado y financiado por el IPCE del Ministerio de Cultura español durante los años 2008 a 2012, y coordinado por la Universidad de Cádiz y la Università Ca' Foscari Venezia, dirigido por los Dres. D. Bernal-Casasola y D. Cottica, cuyos resultados se encuentran actualmente en fase de estudio y publicación.

\section{BIBLIOGRAFÍA}

A.O.A.C. (1990): Official Methods of Analysis. Association of Official Analytical Chemistry, Washington, D.C., $15^{\mathrm{a}}$ edición.

Bernal-Casasola, D.; Cottica, D. y Zaccaria, A. (2009): "El garum de Pompeya y Herculano (2008-2012). Síntesis de la primera campaña del proyecto hispano-italiano". Informes y Trabajos 3: 125-137.

Bernal-Casasola, D.; Cottica, D. y Zaccaria, A. (2010): "Pesca y Garum en Pompeya y Herculano. Síntesis de la segunda campaña del proyecto de investigación (2009)". Informes y Trabajos 5: 138-149.

Bernal, D.; Cottica, D.; Zaccaria, A.; Arévalo, A.; Bermejo, J.; Bustamante, M.; Carrera, C.; DomínguezBella, S.; García-Vargas, E.; Lagóstena, J.; Lara, M.; Lorenzo, L.; Romero, A.; Sáez, A. M. y Villada, F. (2009): Memoria definitiva del Proyecto "El Garum de Pompeya y Herculano. Explotación de los recursos del mar en el ámbito vesubiano", II Campaña (2009). Original depositado en el Instituto del Patrimonio Cultural de España del Ministerio de Cultura y en las Universidades de Cádiz y Venecia.

Bernal, D.; Cottica, D.; Bustamante, M.; Díaz, J. J.; Expósito, J. A.; García, E.; Gómez, A.; Landi, S.; Lara, M.; Lorenzo, L.; Marlasca, R.; Riquelme, J. A.; Rodríguez, C. G.; Sáez, A. M.; Vargas, J. y Verdugo, J. (2013): "Pesca y garum en Pompeya y Herculano. Cuarta campaña arqueológica (2011)". Informes y Trabajos 9: 322-339.

Bernal, D. y Sáez, A. M. (2006): "Infundibula gaditana. Acerca de los vasos troncocónicos perforados para filtar garum y otros usos industriales en la bahía de Cádiz". Romula 5: 167-218.

Betancort, J. R. (1997): Determinación de aminoácidos por cromatografía líquida de alta resolución. Estudio cualitativo y cuantitativo de aminoácidos libres en algas y su variación por condiciones de cultivo. Tesis doctoral, Universidad de Las Palmas de Gran Canaria, Departamento de Química. Inédita.

Curtis, R. I. (1979): "The Garum Shop of Pompei". Cronache Pompeiane 5: 5-23.

Curtis, R. I. (1983): "In defense of garum". The Classical Journal 78: 232-240

Curtis, R. I. (1991): Garum and Salsamenta. Production and Commerce in Materia Medica. Leiden, Brill.

Curtis, R. I. (2001): Ancient food technology. Leiden-Boston, Brill.

Dallongeville, S.; Garnier, N.; Bernal, D.; Bonifay, M.; Rolando, C. y Tokarski, C. (2010): "Dealing with the identification of protein species in ancient 
amphorae". Analytical and Bioanalytical Chemistry: 1-11.

Étienne, R. y Mayet, F. (2002): Salaisons et sauces de poisson hispaniques. París, Diffusion E. de Boccard.

García Vargas, E. y Bernal Casasola, D. (2009): "Roma y la producción de garum y salsamenta en la costa meridional de Hispania. Estado actual de la investigación”, en D. Bernal (ed.), Arqueología de la pesca en el Estrecho de Gibraltar. De la Prehistoria al fin del mundo Antiguo: 133-181. Cádiz, Diputación Provincial.

Gargilius Marcialis: Confectio liquaminis quod omogarum vocant, Cod. Sang. 752 y 899. http://www.ecodices.unifr.ch/en/csg/0752/130/medium, http:// www.e-codices.unifr.ch/en/csg/0899/139/medium (consulta 10 de junio de 2012).

Gomez, J.G.C. (2000): Produção por Pseudomonas sp de polihidroxialcanoatos contendo monómeros de cadeia média a partir de carboidratos: Avaliação da eficiencia, modificação da composição e obtanção de mutantes. Tese (Doutorado)- Instituto de Ciéncias Biomédicas, Universidade de Sao Paulo, Sao Paulo, Brasil. Inédita.

Grimal, P. y Monod, T. (1952): "Sur la veritable nature du garum". Revue des Études Anciennnes 54: 26-38.

Jardin, C. (1961): "Garum et sauces de poisson de l'antiquité”. Rivista di Studi Liguri XXVII(1-4): 70-96.

Köhler, M. (1832): “Tárichos ou recherches sur l'histoire et les antiquités des pêcheries de la Russie Méridionale". Mémoires de l'Academie Impériale de Sciences de St. Petersburg, vol. 1, 6e série, t.1: 347-490.

Lowe, B. (2009): "Fish sauces in ancient Rome: a historic perspective on the use of free glutamates to enhance flavor", en Abstracts Presented at the Pangborn Conference, ESN Workshop: Characteristics of memory for foods: consequences for sensory and consumer science, Florencia. Inédito.
Millán León, J. (2001): “A propósito de la marca Soc y en torno al Garum Sociorum". Habis 32: 171-184.

Ribechini, E.; Colombini, M. P.; Giachi, G.; Modugno, F. y Pallecchi, P. (2009): "A multi-analytical approach for the characterization of commodities in a ceramic jar from Antinoe (Egypt)". Archaeometry 51(3): 480-494.

Rose, V. (1874): "Aringus, der Hering". Hermes 8(2): 226-227.

Smriga, M.; Mizukoshi, T.; Iwahata, D.; Eto, S.; Miyano, H.; Kimura, T. y Curtis, R. I. (2010): “Amino acids and minerals in ancient remnants of fish sauce (garum) sampled in the "Garum Shop" of Pompeii, Italy". Journal of Food Composition and Analysis 23: 442-446. doi: http://dx.doi.org/10.1016/j. jfca.2010.03.005.

Sternberg, M. (2007): "Salaisons et sauces de poisson. Production et produits", en Sternberg, M. (ed.), $G a-$ rum et pissalat. De la pêche à la table. Mémoire d'une tradition: 25-39. Antibes, Musée Archéologique.

Suzzane, S. (2007): Análisis de los alimentos. Manual de laboratorio. Zaragoza, Acribia.

Toppe, J.; Albrektsen, S.; Hope, B. y Aksnes, A. (2007): "Chemical composition, mineral content and amino acid profiles in bones from various fish species". Comparative Biochemistry and Physiology, Part B. 146: 395-401. http://dx.doi.org/10.1016/j. cbpb.2006.11.020.

Van Neer, W.; Ervynck, A. y Monsieur, P. (2010): "Fish bones and amphorae: evidence for the production and consumption of salted fish products outside the Mediterranean region". Journal of Roman Archaeology 23: 161-195.

Van Neer, W. y Parker, S. Th. (2008): "First archaeozoological evidence for haimation, the 'invisible' garum". Journal of Archaeological Science 35: 1821-1827. hdl.handle.net/10.1016/j. jas.2007.11.021.

\section{APÉNDICE}

\section{Confectio liquaminis quod omogarum vocant}

Medicina ex oleribus et pomis (Cod. Sang. 752 y 899).

Publicado inicialmente en V. Rose (1874): “Aringus, der Hering”. Hermes 8.2: 226-227.

Capiuntur pisces natura pingues, ut sunt salmones et anguillae et alause et sardine vel aringi, et fit ex eis atque ex herbis odoratis aridis cum sale compositio talis. Preparatur vas bene solidum ac bene picatum, capax trior vel quattuor modiorum, sumunturque herbe aride bene olentes, tam de orto quam de agro (...) et ex his in fundo vasis primus ordum constetrmitur. Tum ex piscibus, si minores fuerint integris, si maiores in frustra 
concisis alter ordo componitis. Super hunc tertius ordo salis binos digitos altus adicitur. Atque in hunc modum bis tribus herbarum et piscium salisque ordinibus supra inuicem alternantibus vas et usque ad sumitatem implendum, tum addito operculo claudendum atque ita per dies septem dimittendum quibus transactis per continuos viginti dies cotidie bis vel ter palo ligneo in modum remi formato compositio ista usque ad fundum est commovenda. Quibus expletis liquor qui de hac compositione defluxit colligitur atque in hunc modum ex eo liquamen vel omogarum conficitur. Summuntur huius liquoris sextarii duo et cum dimidio boni vini sextario commiscentur, tum quattuor herbarum aridarum singuli manipuli in hanc mixtura coiciuntur (...) Haec minute contrita eidem liquori permiscentu. Tum vel in ferreo vel in ereo vase compositio haec tam diu coquenda est quosque ad unius sextarii mensuram perveniat. Prius tamen quam percoquatur mellis despumati selibram in eam adici oportet. Quae cum percocta fuerit more potionum per sacculumcolari debet usque ad claritatem, fervens tamen sacco infundenda est. Eliquata vero et refrigerate in vase bene picato servatur ad obsonia condienda. 